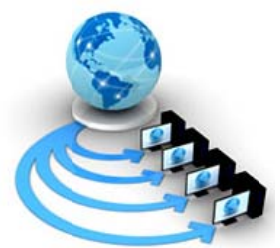

\title{
COMPARATIVE ANALYSIS OF NON CO OPERATIVE AND CO OPERATIVE ROUTING PROTOCOLS IN UNDERWATER COMMUNICATION NETWORKS
}

\author{
Seema Choudhary \\ M.E. Scholar \\ Computer Science Department, \\ Dr. N.C. Barwar \\ Professor \\ M.B.M. Engineering College, J.N.V. University, \\ Jodhpur, Rajasthan, India \\ M.B.M. Engineering College, J.N.V. University, \\ Jodhpur, Rajasthan, India
}

\begin{abstract}
Underwater communication networks (UWCNs) include sensors and autonomous underwater vehicles (AUVs) that interact to perform specific applications such as underwater monitoring. Some of the applications like Mission critical applications require reliability and network efficiency in Underwater Communication Networks, so for coordination and data forwarding between sensor nodes cooperative communication routing protocols are developed that enhances network efficiency. Cooperation is added to the existing non-cooperative routing protocol at network layer that perform better in terms of reliability and throughput. This paper focuses on evaluation of non co operative routing protocols DBR (Depth Based Routing Protocol), EEDBR (Energy Efficient Depth Based Routing Protocol) and cooperative routing protocols Co DBR, Co EEDBR in terms of packet delivery ratio, end to end delay, throughput and energy consumption. NS2 simulator judge the performance of these protocols. Simulation results show that Cooperative Routing Protocols outperforms than Non Cooperative Routing Protocols.
\end{abstract}

\section{Keywords: DBR, EEDBR, Co DBR, Co EEDBR}

\section{INTRODUCTION}

Underwater communication has become an important data transmission technology that is widely used in various ocean applications such as oil/gas spill monitoring, off-shore oil industry, pollution monitoring in environmental, disaster prevention, submarine detection and surveying sea floor for detection of objects and search for new resources. Underwater communication networks consist of a variable number of sensors equipped with transducer, buoys, surface sink, stations [3] and autonomous underwater vehicles (AUVs) that interact each other for a particular application over given area. Acoustic signal is best suited for underwater communication $[1,4]$.

Routing in underwater sensor networks (UWCNs) is very challenging because of unique characteristics of UWCNs and also UWCNs have very dynamic topology as sensors move with water currents. A number of routing protocols have been proposed to deal with the challenging problem in UWCNs. Localization process which requires full dimensional location of sensor nodes is used in most of the routing protocol. That is yet major challenging issue to be solved in UWCNs. Recently, many geographical routing protocols have been proposed for UWCNs, which can be classified into two sections, localization-based and localization-free routing protocols [2].In this paper localization free routing protocols DBR, EEDBR, Co DBR and Co EEDBR based on co operation are considered for further analysis. DBR [8] is a non-cooperative receiver based routing protocol in which the routing is depends only on depth of the sensor node. Drawbacks of DBR is improved in the EEDBR, where depth from sink as well as residual energy of sensor nodes is used to select the candidate forwarder to achieve load balancing. Co DBR overcomes the problems associated with DBR and EEDBR by using cooperative diversity [14]. Co DBR transmits the packet to the destination thru two relay nodes [13] .The relay nodes selects on the basis of minimum depth and they cooperatively deliver the data to the sink. The packet delivery ratio will be increases because in case of any link failure at least one link is capable of delivering the data successfully to the destination. Co DBR consumes three times more transmission energy than DBR because it uses source node and two relay nodes to transmit data, so to achieve reliability energy will be compromised also every time it consider the lower depth nodes. To handle problems associated with all these three protocols, a new cooperative routing protocol Co EEDBR [9] is introduced. Co EEDBR route the data through the UW network nodes with reduced path-loss [15] over the channel [14]. Advantages of singlehop and multi-hop are taken into account as well. Relaying techniques used are AF and FR.

\section{ROUTING PROTOCOLS IN UWCN}

Recently, many geographical routing protocols have been proposed for UWCNs, which can be classified into two sections, localization-based and localization-free routing protocols. In this paper the localization free routing protocols are considered for further analysis.

\subsection{DBR (Depth Based Routing) Protocol}

DBR is a non-cooperative receiver based routing protocol in which source node broadcasts its data to all its neighbors [5]. DBR transmits data packets greedily towards the water surface where data sinks are situated and the transmission is based on the depth information of each sensor. In DBR After receiving a packet node holds the packet for certain amount of time called holding time and then transmits the packet if the depth of the node is smaller than the depth of previous sender that is embedded in the packet. Otherwise, it discards the packet $[5,10,11]$. Holding time depends on the 
depth of the node from the sink. There are multiple data sinks deployed at the water as in multiple-sink network architecture so packets reach any of the sinks are treated as successfully delivered to the destination. To summarize, the main advantages of DBR are as follows. 1) No need of full dimensional location information. 2) Dynamic topology handled easily with good energy efficiency. 3) Without introducing extra cost DBR exploit the benefits of multiplesink network architecture [3].

\subsection{EEDBR (Energy-Efficient Depth-Based Routing Protocol)}

In DBR smaller depth nodes participates in forwarding the data packet, most of the time. As a result the nodes having smaller depth die earlier than the other nodes in the network which create the routing holes all over the network, which severely affects the network lifetime. Furthermore, in DBR, the number of nodes may have similar depth and holding times. All these nodes forward packet at the same time so, a number of redundant packets will be forwarded that increase energy consumption. DBR don't have the concept of residual energy so energy balancing between sensor nodes is difficult [6].To solve these problems EEDBR protocol is used that consider residual energy and balance energy consumption between nodes. It takes into account depth and residual energy of nodes to forward the data [11]. During packet forwarding in EEDBR the packet is transmitted by some intermediate nodes that are nearer to the water surface or have lower depth and more residual energy than other nodes. In EEDBR when a node receives a packet it compares its depth with previous hop, if depth of the current node is less than previous hop then the current node holds the packet for a certain amount of time called holding time before forwarding. Residual energy is the key factor in determining holding time and its inversely proportional to the residual energy. In this way high residual energy nodes having short holding time and always transmit data first. The energy balancing is achieved in this way improves the network life time $[6,7]$.

\subsection{Co DBR (Cooperative Routing Protocol)}

Co DBR uses cooperative diversity to solve the problems associated with DBR and EEDBR. Co DBR find three nodes based on depth and select minimum depth node as a sender [12]. Data is forwarded from source to sink thru the path that established priory. [8]. After receiving the data relay nodes retransmit the same data using AF (Ampliy and Forward) scheme. Amplification is needed because path loss, fading and noise weaken the signal. Destination receives three faded copies of signal from source and relay nodes.

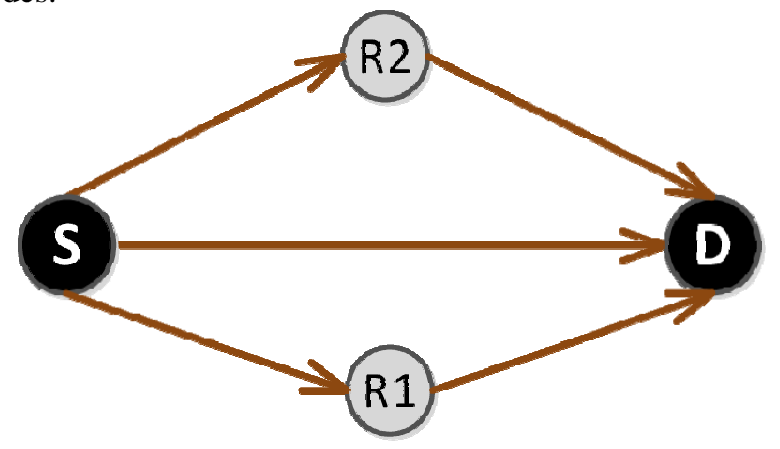

Figure 1: Co DBR System Model

These three copies of data are combined using MRC technique [14]. Now at the destination BER is calculated and checks against the threshold $\mathrm{T}$. $\mathrm{T}$ is the maximum allowable error rate in data. Packet is accepted only if BER is less than or equal to T, otherwise discarded. Co DBR has multi-hop path so this process is repeated at each next hop destination till the sink is reached.

\subsection{Co EEDBR (Cooperative Energy Efficient Depth Based Routing) Protocol}

Co DBR transmits the packet to the destination thru two relay nodes. In this way Co DBR consumes three times more transmission energy than DBR because it uses source node and two relay nodes to transmit data, so to achieve reliability energy will be compromised also every time it consider the lower depth nodes. To solve these problems a new co operative routing protocol Co EEDBR [9] is introduced. In Co EEDBR data is transmitted from source node to sink where, source node transmits its data to master and cooperative nodes. At destination BER is calculated. If $\mathrm{BER}$ is less than or equal to maximum allowable BER, E, data packet is accepted and master node sends ACK signal to relay nodes. When relay nodes receive the ACK signal, relay nodes discard the data. Otherwise, if BER is greater than E, a negative acknowledgement signal, NACK, is sent by master node to relay nodes. Now when there is NACK the master node receive data from relay nodes. Data is combined using MRC techniques and again BER is calculated and check with threshold. If BER is less than or equal to E, data packet is accepted and ACK signal is sent to relay nodes and when relay nodes receive the ACK signal they discard the data. In Co EEDBR, after the failure of two retransmissions, data packet is dropped.

\section{NS2 SIMULATION}

NS2 is an object-oriented, discrete event driven network simulator, which interprets object oriented $\mathrm{Tcl}(\mathrm{OTcl})$ using object libraries provided by Simulator. It is developed in $\mathrm{C}++$ as back end and OTcl as front end.

NS2 is used for Simulation Mobility scenarios are generated by using a random way point model by varying 10 to 100 nodes moving in simulation area of $1500 \mathrm{~m} \times 1500 \mathrm{~m}$. We have used the following parameters.

Table 1: Simulation metrices

\begin{tabular}{|c|c|}
\hline $\begin{array}{c}\text { SIMULATION } \\
\text { SOFTWARE }\end{array}$ & NS2.30 \\
\hline NETWORK SIZE & $1000 \mathrm{M} \mathrm{X1000} \mathrm{M}$ \\
\hline NO OF NODES & $5,10, \ldots,, 100$ \\
\hline INITIAL ENERGY & $50 \mathrm{~J}$ \\
\hline PACKET SIZE & 812 BITS \\
\hline TX POWER & 0.75 \\
\hline RX POWER & 0.75 \\
\hline NO OF SINKS & 1 \\
\hline
\end{tabular}




\subsection{Performance Metrics}

The simulation of Non Cooperative and Cooperative routing protocol is done using following performance parameters:

1) Packet Delivery Ratio: The ratio between the amount of incoming data packets and actually received data packets.

2) End to End Delay: Represents average end-to-end delay and specify how long it took for a packet to travel from the source to the application layer of the destination.

3) Throughput: It represents the total number of bits forwarded to higher layers per second. It is measured in bps.

4) Total Energy consumption: Energy needed to send, receive and forward the data.

Figure 2 shows the PDR analysis for DBR, EEDBR, Co DBR and Co EEDBR with varying number of nodes in the simulation. It is observed from the graph that Co EEDBR performs better than other protocols. Co-EEDBR and CoDBR protocols achieve greater PDR in comparison to DBR and EEDBR. Higher packet loss in DBR is due to inconsideration of channel estimation. Cooperative schemes improve the packet delivery by transmitting packets on multiple paths and combining these different copies of packet at receiver end. When all nodes are alive, Co DBR has double PDR than DBR. As nodes start to die, PDR starts decreasing and it's nearer to DBR because less number of packets sent to sink and more packets are dropped. CoEEDBR outperforms Co-DBR, DBR and EEDBR due to less delay in data delivery. PDR decreases with increase in network density.

Figure 3 shows the throughput analysis for DBR, EEDBR, Co DBR and Co EEDBR protocol with varying number of nodes in the simulation. It is observed from the graph that Co EEDBR outperforms Co DBR, DBR and EEDBR due to less delay in data delivery and balance load on nodes to increase the network life time that increases throughput.

Figure 4 shows the energy consumption analysis for DBR, EEDBR, Co DBR and Co EEDBR protocol with varying number of nodes in the simulation. It is observed from the graph that as number of nodes increases, energy consumption in all the protocols increases. Figure 4 depicts that EE-DBR consume less energy than DBR because excessive number of nodes involve in forwarding the data packet, redundant packet transmissions due to poor link consideration and also the calculation of holding time for a packet at each node in DBR. Co DBR is consuming more energy than DBR because Co DBR utilizes source node along-with two relay nodes to transmit data to the next-hop. Co EEDBR consumes less energy than Co DBR as Co EEDBR employ energy balancing techniques to reduce energy consumption.

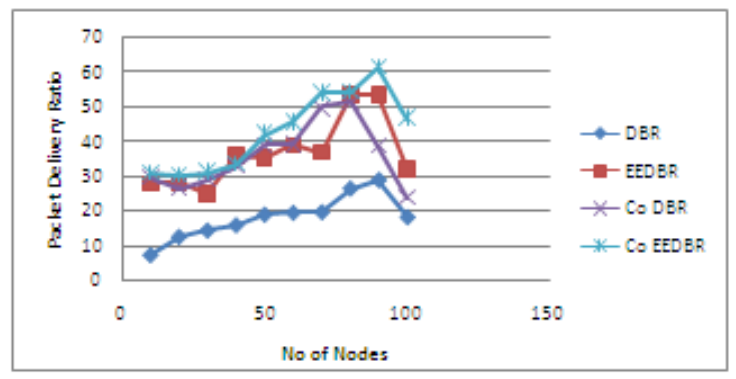

Figure 2: PDR of DBR, EEDBR, Co DBR and Co EEDBR
Figure 5 shows the delay analysis for DBR, EEDBR, Co DBR and Co EEDBR protocol with varying number of nodes in the simulation. It is observed from the graph that DBR, EEDBR, Co DBR and Co EEDBR has lower end-toend delay in dense network than sparse networks because the number of candidate forwarders is larger in dense network, and it has a higher probability to have an optimal next hop. EE-DBR has a better performance than DBR, Co DBR and Co EEDBR in end- to-end delay. EE-DBR reduces network collision, so end-to-end delay is also reduced because the first node in the list of forwarding nodes, transmit the packet as soon as it receives the packet. Every node in DBR holds the packet for certain amount of time that increases delay.

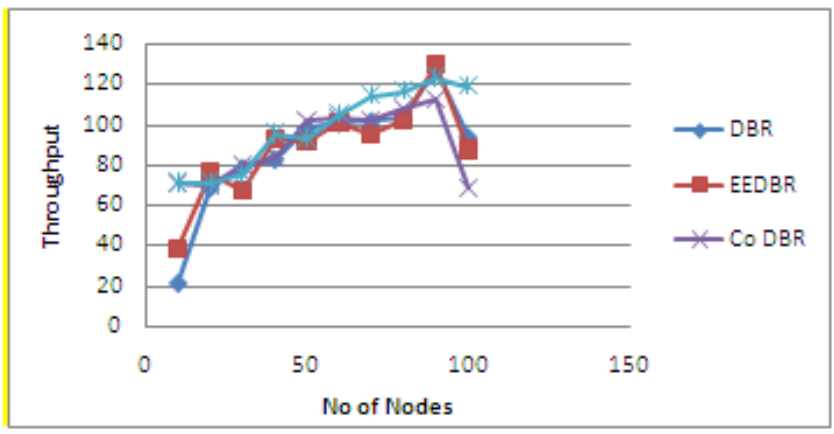

Figure 3: Comparative Throughput of DBR, EEDBR, Co DBR and Co EEDBR

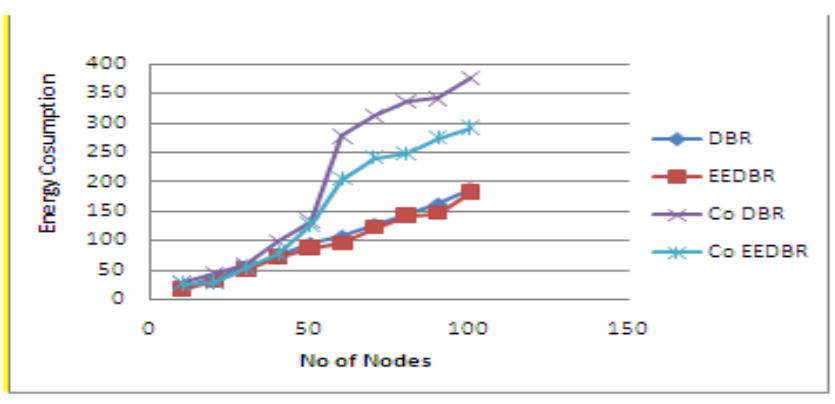

Figure 4: Comparative Energy of DBR, EEDBR, Co DBR and Co EEDBR

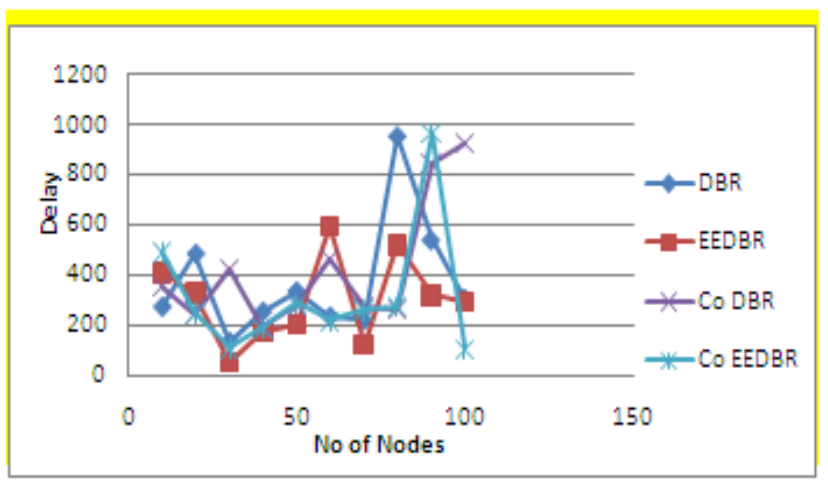

Figure 5: Comparative Delay of DBR, EEDBR, Co DBR and Co EEDBR

\section{CONCLUSION}

In this paper, analysis and evaluation of DBR, EEDBR, Co DBR and Co EEDBR protocols have been considered with respect to different performance parameters such as packet 
delivery ratio, throughput end-to-end delay and energy consumption. It is noted that the performance of Co EEDBR protocol is better as compared to other protocols with varying number of nodes. Co DBR proved to be beneficial for mission critical applications. It has less packet drop and improved throughput compared to DBR in noisy underwater environment. However, it consumes more energy and more end-to-end delay. In energy-sensitive scenarios, like longtime marine data sampling, EE-DBR is a better choice. Co EEDBR is suitable for delay-sensitive applications, but not for reactive applications, flooding-based protocols. In future, better criteria for the selection of relays and destination may be proposed to achieve better load balancing of network.

\section{REFERENCES}

[1] Akyildiz, Ian F., Dario Pompili, and Tommaso Melodia. "Challenges for efficient communication in underwater acoustic sensor networks." ACM Sigbed Review1.2 (2004):3-8.

[2] Kiranmayi, M., and Kathirvel Ayyaswamy. "Underwater Wireless Sensor Networks: Applications, Challenges and Design Issues of the Network Layer-A Review."

[3] Singh, Tanu, and Manu Singh. "UW-Hybrid Architecture of Underwater Acoustic Sensor Networks."

[4] Pompili, Dario, and Ian F. Akyildiz. "Overview of networking protocols for underwater wireless communications." Communications Magazine, IEEE 47.1 (2009): 97-102.

[5] Yan, Hai, Zhijie Shi, and Jun-Hong Cui. "DBR: depth-based routing for underwater sensor networks." NETWORKING 2008 Ad Hoc and Sensor Networks, Wireless Networks, Next Generation Internet (2008): 72-86.

[6] Wahid, Abdul, and Dongkyun Kim. "An energy efficient localization-free routing protocol for underwater wireless sensor networks." International journal of distributed sensor networks 8.4 (2012): 307246.

[7] Najeeb, F., et al. "A new advanced energy efficient routing protocol for UWSNs." J Basic Appl Sci Res 4.2 (2014): 4353.

[8] Nasir, Hina, et al. "CoDBR: cooperative depth based routing for underwater wireless sensor networks." Broadband and Wireless Computing, Communication and Applications (BWCCA), 2014 Ninth International Conference on. IEEE, 2014.

[9] Ahmad, Ashfaq, et al. "On energy efficiency in underwater wireless sensor networks with cooperative routing." Annals of Telecommunications 72.3-4 (2017): 173-188.
[10] Guangzhong, Liu, and Li Zhibin. "Depth-based multi-hop routing protocol for underwater sensor network." Industrial Mechatronics and Automation (ICIMA), 2010 2nd International Conference on. Vol. 2. IEEE, 2010.

[11] Khasawneh, Ahmad, et al. "Next forwarding node selection in underwater wireless sensor networks (UWSNs): Techniques and challenges." Information8.1 (2016): 3.

[12] Mahmood, S., et al. "Forwarding nodes constraint based DBR (CDBR) and EEDBR (CEEDBR) in underwater WSNs." Procedia Computer Science 34 (2014): 228-235.

[13] Javaid, Nadeem, et al. "Cooperative Opportunistic Pressure Based Routing for Underwater Wireless Sensor Networks." Sensors 17.3 (2017): 629.

[14] Tan, Do Duy, Tung Thanh Le, and Dong-Seong Kim. "Distributed cooperative transmission for underwater acoustic sensor networks." Wireless Communications and Networking Conference Workshops (WCNCW), 2013 IEEE. IEEE, 2013.

[15] Al-Dharrab, Suhail, Murat Uysal, and Tolga M. Duman. "Cooperative underwater acoustic communications [Accepted from open call]." IEEE Communications Magazine 51.7 (2013): 146-153.

\section{AUTHOR}
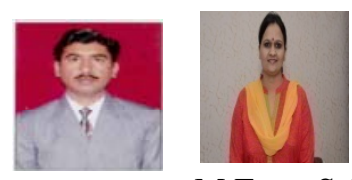

Seema choudhary is currently a M.E. Scholar in Computer Science Engineering at M.B.M. Engineering College. Her field of interest is Computer Network, Database management System etc.

Dr. N.C. Barwar is currently working as Professor in M.B.M Engineering College. He received B.E. from M.N.I.T. Bhopal, M.E. in Digital Communication from M.B.M. Engineering College and $\mathrm{PhD}$ from J.N.V. University. He has published more than 40 papers in national and international conferences and journals and having teaching experience of more than 20 years at PG and UG levels. His field of interest is Computer Networks, Multimedia, VOD, and Information theory etc. 